\title{
A novel High-Speed Electromagnetic Oscillatory Actuator with a Dual Mover for Optical Scanner Applications
}

\author{
Yinggang $\mathrm{Bu}^{1}$, Shintaro Yoshida ${ }^{1}$, Kazuaki Oyaizu ${ }^{1}$, Hideyuki Kinjo ${ }^{1}$, Kaname Inoue ${ }^{1}$, Akihiro Nitta ${ }^{1}$, \\ Tsutomu Mizuno ${ }^{1}$, and Zhihui Duan ${ }^{2}$ \\ ${ }^{1}$ Faculty of Engineering, Shinshu University, Nagano 380-8553, Japan \\ ${ }^{2}$ Optoelectronics Co., Ltd., Warabi, Saitama 335-0002, Japan
}

In this paper, we propose a high-speed moving magnet-type oscillatory actuator in which a dual-mover structure is designed to reduce magnetic damping force. Both movers have independent resonant frequency, and the actuator is driven at the larger resonant frequency. The smaller mover serves as the optical scanning element and maintains large oscillation amplitude. The larger mover, with moving magnets, serves as the magnetic driving element and maintains relatively smaller amplitude because of the magnetic damping.

Index Terms-Actuator with dual movers, high speed oscillation, magnetic damping, optical scanner.

\section{INTRODUCTION}

$\mathrm{O}^{\mathrm{n}}$ PTICAL scanning actuators are widely employed for laser printing, barcode readers, laser displays, etc. Applications such as laser television and laser printing require scanning frequencies of more than a few thousand to several tens of thousand $\mathrm{Hz}$ [1]-[3]. Typical high-speed optical actuators realize sweeping performance using polygon motors. However, because polygon motors have large sizes and high power consumption, their installation into portable devices is difficult. In addition, it is difficult for polygon motors to achieve scanning frequencies of several tens of thousand $\mathrm{Hz}$ such as those required for laser television [4]. With improvements in semiconductor processes, an actuator with a micro electromechanical system (MEMS) process is becoming practical to simultaneously realize low power and small size [2]. However, MEMS mirrors that generally use piezoelectric or static electric drives require several tens of voltage in their circuitry compared with the desired 3-5 V circuitry design in a portable device [5], [6].

Electromagnetic drives can be controlled at low voltage with large driven force using either a moving coil or a moving magnet. Because the moving coil drive is easily controlled and has a fast response, it is popularly used in both MEMS and general electromagnetic actuators. However, because the power-supplied coil is movable, it is unfavorable for scanning applications with critical reliability requirements. In addition, the moving coil drive requires a large magnet to provide sufficient external magnetic strength to drive the actuator [7].

The moving magnet drive uses a permanent magnet instead of a coil in movable element. Without a movable electrical power line, the actuator structure can be relatively simple and potentially has higher reliability than the moving coil drive. In a typical moving magnet structure, the yoke and the magnet are separated by an air gap. The yoke directly faces the magnet to increase the magnetic density, which increases the force. Therefore, eddy currents in the yoke will be generated by variable magnetic flux between the moving magnet and the

Manuscript received March 7, 2014. Corresponding author: Y.Bu (email: buyinggang@ shinshu-u.ac.jp).

Digital Object Identifier inserted by IEEE yoke, resulting in the magnetic damping force. This damping force is not negligible when the driving frequency is high. In such cases, the driving power will rapidly increase if the same amplitude is retained as in the low driving frequency. Therefore, the conventional structure with a moving magnet drive is not adoptable in a high-frequency actuator.

In a conventional moving magnet structure, a permanent magnet and optical mirror are rigidly installed to the same rotator to construct a mover. Thus, both the magnet and the mirror have the same rotating angle as that of the rotator. Considering this typical moving magnet structure, in this study, we intentionally separated the magnet and the mirror into two rotators to construct dual movers. The first mover is composed of the first rotator and a pair of permanent magnets surrounding the second mover. The second mover is composed of the second rotator, and an optical mirror is located at the center of the first mover. The first mover is driven by an external electromagnetic force with a low resonant frequency. The temporal variable magnetic damping occurs only at the first mover, which produces small vibration energy. Because magnetic damping does not occur in the second mover, this small vibration energy will produce large vibration amplitude if the vibration frequency is at the resonant frequency of the second mover. As verified in this study, the proposed dual-mover structure can significantly reduce the magnetic damping to achieve the large scanning amplitude required for optical scanning.

\section{ACTUATOR STRUCTURE}

Fig. 1(a) shows the basic structure of the proposed moving magnet actuator with dual movers. The actuator substrate is constructed using a single nonmagnetic stainless steel sheet. The substrate is then etched to a planar pattern to form a stator plate and two separate rotator plates. In general, the larger rotator plate has a rectangular frame shape. A pair of parallel edges of Rotator 1 is connected to the stator plate through a planar bridge pattern. This bridge pattern serves as Torsion spring 1 during the oscillation and is the rotation axis of Rotator 1. An additional pair of parallel edges of Rotator 1 is separated from the stator plate by gaps in which each edge has 
(a)
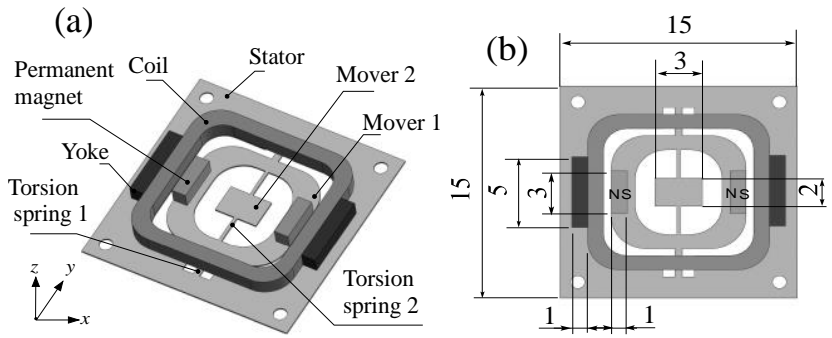

Fig. 1. Basic structure of the proposed actuator in (a) perspective view (b) plane view (unit: $\mathrm{mm}$ ).

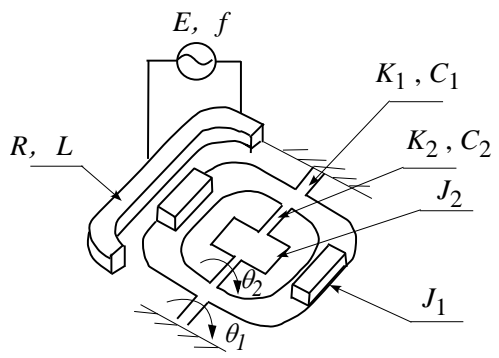

Fig. 2. Dynamic model of the proposed actuator.

an attached permanent magnet. For convenience, Rotator 1, Torsion spring 1 , and the permanent magnets are defined as Mover 1. A loop coil and a pair of the yokes are installed on the stator plate. The yoke pairs face the magnet pairs with the loop coil across the middle. The smaller rotator is a rectangular plate shape and is located at the center of Rotator 1. Rotator 2 is connected to the edges of Rotator 1 through a second planar bridge pattern along the same extension direction as Torsion spring 1 and serves as Torsion spring 2 during the oscillation. Rotator 2 and Torsion spring 2 are defined as Mover 2. The surface of Rotator 2 is optically polished as the reflective mirror.

Because Mover 2 is composed of nonmagnetic stainless steel, it is uninfluenced by the magnetic field introduced by the yokes, coil, and the magnets. Except air resistance, there is no direct damping of the external magnetic force to the oscillating movement of Mover 2. Because the temporal variable flux of the moving magnet on Mover 1 will introduce damping in the yokes to affect the magnetic driving efficiency, we maintained its movement at relatively smaller amplitude. In the next section, we will explain how Mover 1 and 2 work as an entire actuator without the influence of magnetic damping. Fig. 1 (b) shows the dimensions of each element in the proposed actuator. On the basis of these dimensions, we will discuss the principle in the following section.

\section{DYNAMIC MODEL AND ANALYSIS}

First, we analyzed the static mechanical model of the actuator structure to realize large oscillating amplitude on Mover 2 and small amplitude on Mover 1. Fig. 2 shows the illustration of the mechanical model. As indicated from the introduction of the actuator structure, the movements of Mover 1 and 2 can be simplified as a one-dimensional rotating

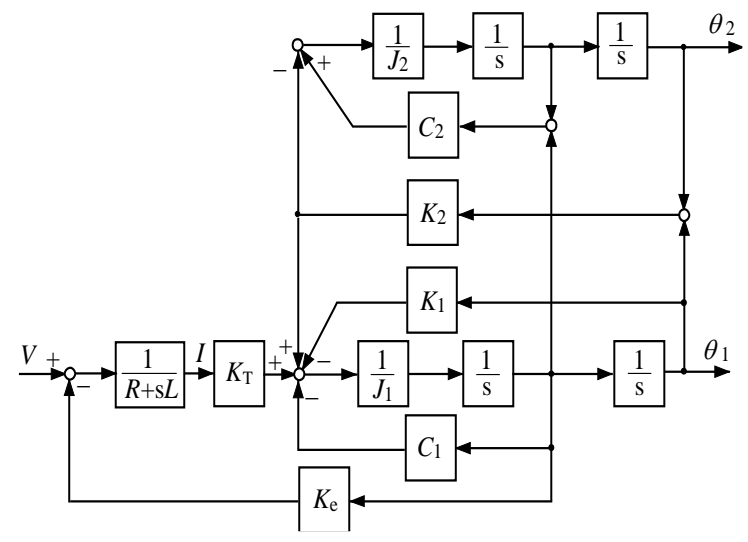

Fig. 3. Dynamic block model of the proposed actuator with dual movers.

TABLE I

ANALYSIS CONDITIONS

\begin{tabular}{llcr}
\hline \hline \multirow{2}{*}{ Symbol } & \multicolumn{1}{c}{ Quantity } & Unit & \multicolumn{2}{c}{ Numerical value } \\
\hline$J_{1}$ & Inertial moment of & \multirow{2}{*}{$\mathrm{mm}^{2}$} & 0.886 \\
$J_{2}$ & mover & $\mathrm{mN} \cdot \mathrm{m} / \mathrm{A}$ & 0.00416 \\
$K_{\mathrm{T}}$ & Torque constant & $\mu \mathrm{N} \cdot \mathrm{m} / \mathrm{rad}$ & 0.28 \\
$K_{1}$ & Spring constant & $\Omega$ & 9055 \\
$K_{2}$ & DC resistance of & $\Omega 004$ \\
$R$ & coil & $\mathrm{mH}$ & 222 \\
& Inductance of coil & & 3 \\
$C_{1}$ & Damping constant & $\mu \mathrm{N} \cdot \mathrm{s} / \mathrm{m} \cdot \mathrm{rad}$ & 0.202 \\
$C_{2}$ & & & 0.0018 \\
\hline
\end{tabular}

oscillation model. The resonant frequencies, $f_{01}$ and $f_{02}$, are determined by their own inertia moments and spring constants. The movement can be expressed as the following functions.

$$
\begin{array}{r}
J_{1} \ddot{\theta}_{1}=-C_{2}\left(\dot{\theta}_{1}-\dot{\theta}_{2}\right)-C_{1} \dot{\theta}_{1}-K_{1} \theta_{1}-K_{2}\left(\theta_{1}-\theta_{2}\right)+T \\
J_{2} \ddot{\theta}_{2}=C_{2}\left(\dot{\theta}_{1}-\dot{\theta}_{2}\right)+K_{2}\left(\theta_{1}-\theta_{2}\right) \quad(\mathrm{N} \cdot \mathrm{m})
\end{array}
$$

From the dynamic block diagram derived from the abovementioned static mechanical model, we calculated the rotation angles $\theta_{1}$ and $\theta_{2}$ of the two movers using Matlab simulink. Compared with the resistance of the loop coil, its inductance was only $3 \mathrm{mH}$, even at $5 \mathrm{kHz}$; therefore, we ignored the inductance in simulink calculation.

Table I shows the perimeter values used for simulink. By finite element method (FEM) calculation, we obtained $J_{1}$ and $J_{2}$ as the values of inertial moments for Mover 1 and 2, respectively, and the values of torque constant $K_{\mathrm{T}}$ and spring constants $K_{1}$ and $K_{2}$, respectively. For the value of damping constants $C_{1}$ and $C_{2}$, we measured the data on the basis of the manufactured sample. On the basis of the diagram in Fig. 3 and the perimeter values in Table I, we calculated the log ratio of the rotation angle to driven voltage of Mover 1 and 2, as shown in Fig. 4. The log ratio of the rotation angle to driven voltage is expressed as the following relationship: 


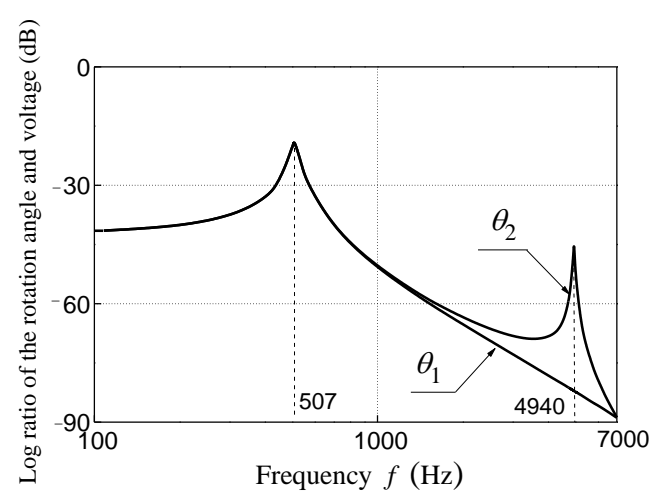

Fig. 4. Calculated log ratio of the rotation angle to driven voltage of Mover 1 and Mover 2.

$$
G=20 \log \frac{\theta}{V}
$$

As indicated in Fig. 4, the actuator has two different resonant frequencies. One is at $f_{01}=507 \mathrm{~Hz}$, and the other is at $f_{02}=4940 \mathrm{~Hz}$, which is independently determined by Mover 1 and 2. As shown in Fig. 4, the rotation angles $\theta_{1}$ and $\theta_{2}$ have close values at a relatively low frequency at $f_{01}=507 \mathrm{~Hz}$, indicating that Mover 2 oscillated at the same performance as that of Mover 1. On the contrary, at the relatively high frequency $f_{02}=4940 \mathrm{~Hz}$, the rotation angles $\theta_{1}$ and $\theta_{2}$ are separated from each other. Because this frequency point is far from the resonant frequency of Mover 1, Mover 1 does not show obvious resonant movement. Compared with Mover 1, which includes only one rotation peak amplitude, Mover 2 followed up with the resonant oscillation of Mover 1 to obtain the first amplitude peak at $507 \mathrm{~Hz}$, and then entered its own resonant oscillation at $4940 \mathrm{~Hz}$. That is, if the actuator has a driving frequency of $4940 \mathrm{~Hz}$, although the pair of moving magnets results in the damping of the magnetic field at Mover 1 and further results in the damping of the oscillation amplitude of Mover 1, it will not influence the resonant oscillation performance of Mover 2. As long as we design the optical scanning element of Mover 2, large optical scanning amplitude can still be realized at high frequency, which is exactly what we expected in a moving magnet actuator.

\section{EXPERIMENTAL EVALUATION}

Fig. 5 shows a photograph of the prototype actuator based on the design of Fig. 1. The actuator substrate is a stainless steel sheet of $0.1 \mathrm{~mm}$ thickness. We slightly expanded the dimension of the loop coil at the location of Torsion spring 1 to avoid an electrical current short between Torsion spring 1 and the loop coil. The rectangular surface of Mover 2 was polished as an optical mirror to reflect the incident laser beam.

Fig. 6 shows the experimental block for measuring oscillation performance. A sinusoidal voltage signal was generated by an oscillator and was amplified to $V_{\text {peak }}=3.0 \mathrm{~V}$ by an amplifier to drive the loop coil.

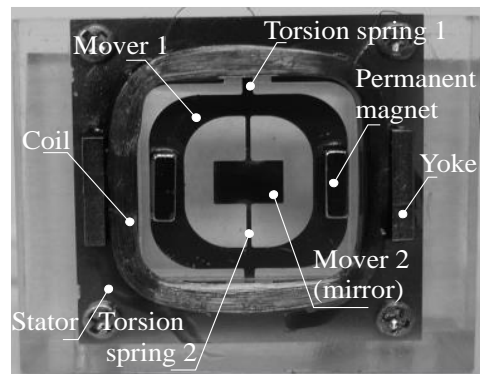

Fig. 5. Photograph of prototype actuator.

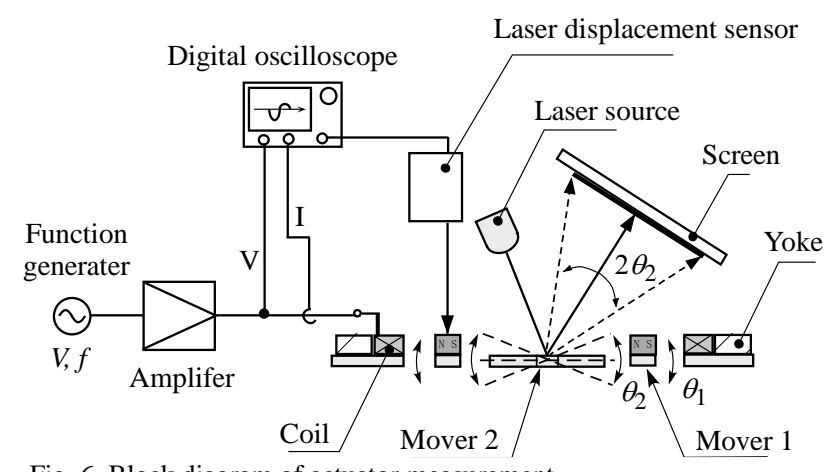

Fig. 6. Block diagram of actuator measurement.

While tuning the frequency of the sinusoidal signal from 100 $\mathrm{Hz}$ to $7000 \mathrm{~Hz}$, we measured the rotation angles $\theta_{1}$ and $\theta_{2}$, respectively. The rotation angle $\theta_{1}$ was measured by a laser displacement sensor, and $\theta_{2}$ was determined by measuring the scan line on the screen reflected from the optical surface of Mover 2.

Fig. 7 shows the measurement results. When driven at the resonant frequency $f_{01}=450.7 \mathrm{~Hz}$ of Mover 1 , both rotation angle (full angle at the maximal amplitude) of Mover 1 and 2 were $\theta_{1}=\theta_{2}=21^{\circ}$. When driven at the resonant frequency $f_{02}$ $=4695 \mathrm{~Hz}$, compared with the rotation angle $\theta_{1}=0.2^{\circ}$ of Mover 1 , the rotation angle of Mover 2 became $\theta_{2}=4.2^{\circ}$, which is approximately 20 times larger than the rotation angle of Mover 1.

Fig. 8 shows an enlarged view of the rotation angle characteristic near the second resonant frequency of $4695 \mathrm{~Hz}$. As we predicted by simulink calculation at this frequency range, compared with the rotation angle $\theta_{2}=4.2^{\circ}$ of Mover 2 determined by resonance, Mover 1 showed only a very small oscillation with the rotation angle $\theta_{1}=0.2^{\circ}$ because its resonant frequency is far from this range. In addition, the experimental results showed a difference of 20 times in rotation angles $\theta_{1}$ and $\theta_{2}$, which exactly matched our simulation results. Therefore, to realize a large amplitude $\theta_{2}=$ $4.2^{\circ}$ of Mover 2 at such a high frequency of $4695 \mathrm{~Hz}$, it is not necessary to directly apply such driven amplitude on Mover 2. Since drive Mover 1 at only $1 / 20^{\text {th }}$ amplitude, the energy loss result of the attenuation of magnetic field can be suppressed to an acceptable extent. 


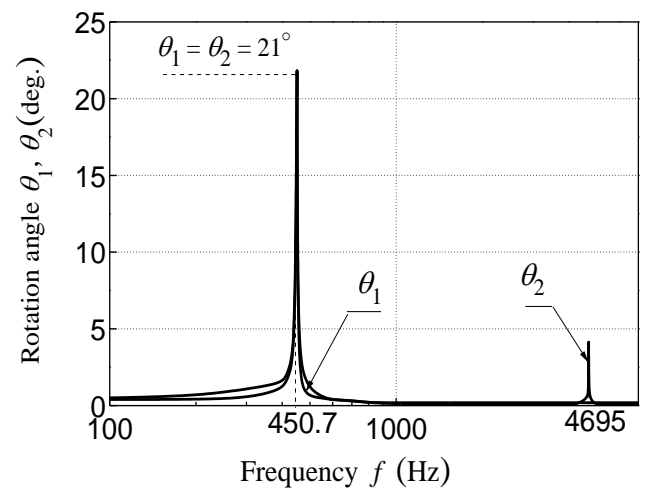

Fig. 7. Rotation angles vs. frequency.

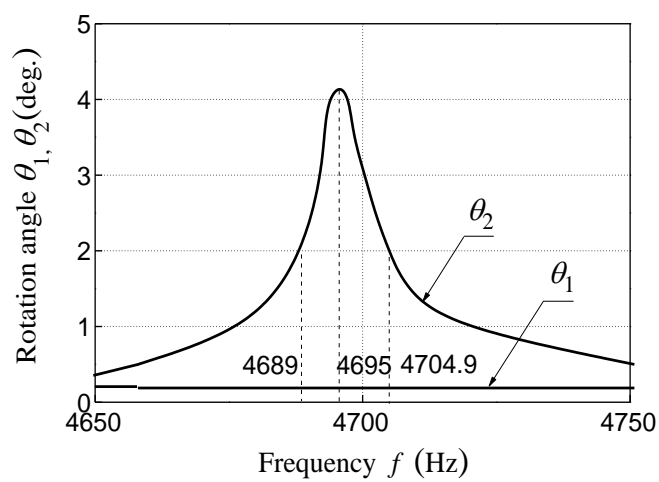

Fig. 8. Rotation angles vs. frequency (enlargement is from $4650 \mathrm{~Hz}$ to 4750 $\mathrm{Hz})$.

Through the resonant characteristics shown in Fig. 7 and Fig. 8 , we can derive the damping coefficient $C$ of Mover 1 and 2 based on the half value method using the following equation:

$$
C=\frac{4 J \lambda}{T \log _{10} e} \quad(\mathrm{~N} \cdot \mathrm{s} / \mathrm{m} \cdot \mathrm{rad})
$$

where $T$ is the period. The logarithmic damping ratio $\lambda$ can be expressed as

$$
\begin{gathered}
\lambda=2 \pi \zeta \\
\zeta=\frac{\Delta f}{2 f_{0}}
\end{gathered}
$$

The damping coefficients of Mover 1 and 2 were calculated as $C_{1}=2.02 \times 10^{-7} \mathrm{~N} \cdot \mathrm{s} / \mathrm{m} \cdot \mathrm{rad}$ and $C_{2}=1.8 \times 10^{-9} \mathrm{~N} \cdot \mathrm{s} / \mathrm{m} \cdot \mathrm{rad}$, respectively. Because $C_{1}$ includes the influence of magnetic damping from the magnets, it is 112 times larger than $C_{2}$. Thus, we can observe that the optimization of the low oscillation amplitude of Mover 1 plays an important role in suppressing the energy loss.

We measured the power consumption of the manufactured actuator. Fig. 9 shows the relationship between the rotation angle and the power consumption at the first and the second resonant frequencies. To achieve the same rotating angle of $4^{\circ}$, the power consumption was approximately $1.46 \mathrm{~mW}$ when driving the actuator at $f_{01}=450.7 \mathrm{~Hz}$, and it became $15 \mathrm{~mW}$ when driving at $f_{02}=4695 \mathrm{~Hz}$, which is a very small power for a portable scanning device.

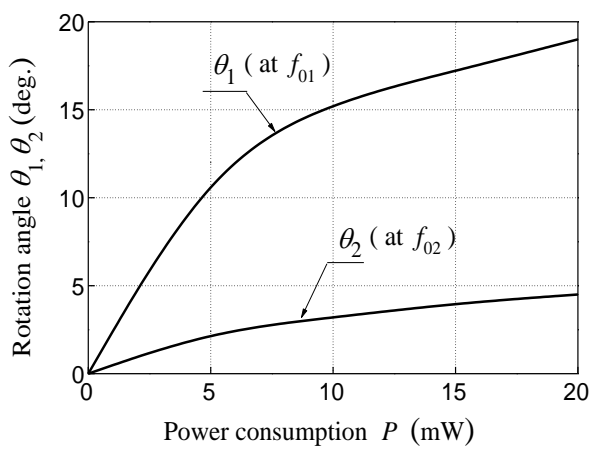

Fig. 9. Rotation angle vs. power consumption at resonant frequency.

Finally, we analyzed the maximum stress in the torsion spring with FEM. The calculation result told it is $336 \mathrm{MPa}$ in Torsion spring 2 when $\theta_{2}=5^{\circ}$ and the maximum stress in Torsion spring 1 is $100 \mathrm{MPa}$ when $\theta_{1}=1^{\circ}$. Compared with the yield strength of more than $500 \mathrm{MPa}$ of the stainless steel SUS301H that we used, it is much smaller level.

\section{CONCLUSIONS}

In this study, we proposed a high-speed moving magnettype oscillatory actuator in which a dual-mover structure is designed to reduce magnetic damping. Both movers have independent resonant frequencies, and the actuator is driven at a large resonant frequency. The experimental results from the prototype actuator indicated that the rotation angle of the larger mover was $\theta_{1}=0.2^{\circ}$ although the rotation angle of the smaller mover was $\theta_{2}=4.2^{\circ}$ when the actuator was driven at the resonant frequency $f_{02}=4695 \mathrm{~Hz}$ of the smaller mover. The rotation angle of the smaller mover for optical scanning was 20 times larger than that of the larger mover. It was proven that when the proposed moving magnet actuator is used, large amplitude can be obtained at a very high frequency. By reducing the driving amplitude, the energy loss resulting from the damping of the magnetic field can be suppressed to an acceptable level.

\section{REFERENCES}

[1] J. Kranert, C. Deter, T. Gessner, and W. Dotzel, "Laser display technology," in Proc. IEEE Micro Electro Mechanical Systems Workshop (MEMS), pp. 99-104, Jan. 1998.

[2] N. Asada, H. Matsuki, K. Minami, and M. Esashi, "Silicon micromachined two-dimensional galvano optical scanner," IEEE Trans. Magn. , vol. 30, no. 6, pp.4647-4649, Nov. 1994.

[3] J. H. Lee, Y.C. Ko, D. H. Kong, J. M. Kim, K. B. Lee and D.Y. Jeon, "Design and fabrication of scanning mirror for laser display," Sens. Actuator A-Phys., vol. 96, pp. 223-230, 2002.

[4] H. Golnabi, "Design and operation of a laser scanning system," Opt. Laser Technol., vol. 32, Issue 4, pp. 267-272, Jun. 2000.

[5] Y. Liu, J. Xu, S. Zhong, and Y. Wu, "Large size MEMS scanning mirror with vertical comb drive for tunable optical filter," Opt. Lasers Eng., vol. 51, Issue 1, pp. 54-60, Jan. 2013.

[6] S. Xiang, S. Chen, X. Wu, D. Xiao, and X. Zheng, "Study on fast linear scanning for a new laser scanner," Opt. Laser Technol., vol. 42, Issue 1, pp. 42-46, Feb. 2010.

[7] L.O.S Ferreira, S. Moehlecke, "A silicon micromechanical galvanometric scanner," Sens. Actuator A-Phys., vol. 73, Issue 3, pp. 252-260, Mar. 1999. 